\title{
No meaningful drug interactions with doravirine, lamivudine and tenofovir disoproxil fumarate co-administration.
}

\author{
Matt S. Anderson \\ Merck \& Co., Inc.; Certara USA, Inc. \\ Jocelyn Gilmartin \\ Merck \& Co., Inc. \\ Li Fan \\ Merck \& Co., Inc. \\ Kollpwa thiseand additional works at: https://jdc.jefferson.edu/petfp \\ 'skark of the InGledical Pharmacology Commons, and the Pharmacy and Pharmaceutical Sciences \\ Dartterors. Kraft

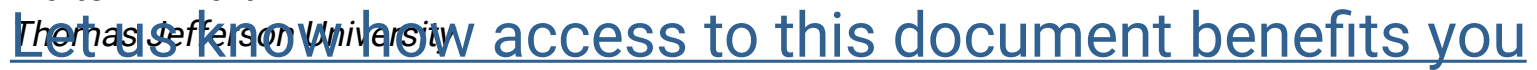

\section{Beconmergerd/Citantional authors}

Anderson, Matt S.; Gilmartin, Jocelyn; Fan, Li; Yee, Ka Lai; Kraft, Walter K.; Triantafyllou, Ilias; Reitmann, Christina; Guo, Ying; Liu, Rachael; and Iwamoto, Marian, "No meaningful drug interactions with doravirine, lamivudine and tenofovir disoproxil fumarate co-administration." (2019). Department of Pharmacology and Experimental Therapeutics Faculty Papers. Paper 110.

https://jdc.jefferson.edu/petfp/110

This Article is brought to you for free and open access by the Jefferson Digital Commons. The Jefferson Digital Commons is a service of Thomas Jefferson University's Center for Teaching and Learning (CTL). The Commons is a showcase for Jefferson books and journals, peer-reviewed scholarly publications, unique historical collections from the University archives, and teaching tools. The Jefferson Digital Commons allows researchers and interested readers anywhere in the world to learn about and keep up to date with Jefferson scholarship. This article has been accepted for inclusion in Department of Pharmacology and Experimental Therapeutics Faculty Papers by an authorized administrator of the Jefferson Digital Commons. For more information, please contact: JeffersonDigitalCommons@jefferson.edu. 


\section{Authors}

Matt S. Anderson, Jocelyn Gilmartin, Li Fan, Ka Lai Yee, Walter K. Kraft, Ilias Triantafyllou, Christina Reitmann, Ying Guo, Rachael Liu, and Marian Iwamoto 


\section{No meaningful drug interactions with doravirine, lamivudine and tenofovir disoproxil fumarate co-administration}

\section{(111/150 characters)}

Matt S Anderson ${ }^{1 \mathrm{a}}$, Jocelyn Gilmartin ${ }^{1}, \mathrm{Li} \mathrm{Fan}^{1}$, Ka Lai Yee ${ }^{1}$, Walter K Kraft ${ }^{2}$, Ilias

Triantafyllou ${ }^{1}$, Christina Reitmann ${ }^{1}$, Ying Guo ${ }^{1}$, Rachael Liu ${ }^{1}$, Marian Iwamoto ${ }^{1}$

${ }^{1}$ Merck \& Co., Inc., Kenilworth, NJ, USA

${ }^{2}$ Thomas Jefferson University, Philadelphia, PA, USA

${ }^{a}$ MSA is a former employee of Merck Sharp \& Dohme Corp., a subsidiary of Merck \& Co., Inc.

Kenilworth, NJ, USA and is now employed by Certara USA, Inc., 100 Overlook Center, Suite

101, Princeton, NJ, USA

Running head (59/75 characters): Doravirine, lamivudine and tenofovir drug-drug interactions

Corresponding author: Marian Iwamoto (marian_iwamoto@merck.com)

Word count: $3,083 / 4,000$

References: $29 / 50$

Total display items: 5/5 [3 tables and 2 figures] 


\section{ABSTRACT [250/250 words]}

Background: Doravirine (DOR) is a novel non-nucleoside reverse transcriptase inhibitor available as a single tablet and a three-drug combination with lamivudine (3TC) and tenofovir disoproxil fumarate (TDF) to treat HIV-1 infection. These analyses assessed pharmacokinetic (PK) interactions with co-administration.

Methods: Two trials were conducted. Study 1: two-period, fixed-sequence; 8 healthy participants; Period 1, DOR $100 \mathrm{mg}$ followed by $\geq 7$-day washout; Period 2, TDF $300 \mathrm{mg}$ once daily for 18 days, co-administration of DOR $100 \mathrm{mg}$ on day 14. Study 2: three-period, crossover, 15 healthy participants; Treatment A, DOR 100 mg; Treatment B, 3TC 300 mg + TDF 300 mg; Treatment C, DOR 100 mg + 3TC 300 mg + TDF 300 mg; $\geq 7$-day washout between periods.

Results: Study 1: geometric mean ratios (GMRs) (90\% confidence interval [CI]) of DOR $\mathrm{AUC}_{0-\infty}$ and $\mathrm{C}_{24 \mathrm{~h}}(\mathrm{DOR}+\mathrm{TDF} / \mathrm{DOR})$ were $0.95(0.80,1.12)$ and $0.94(0.78,1.12)$, respectively. Study 2: GMRs $(90 \% \mathrm{CI})$ of DOR $\mathrm{AUC}_{0-\infty}$ and $\mathrm{C}_{24 \mathrm{~h}}(\mathrm{DOR}+3 \mathrm{TC}+\mathrm{TDF} / \mathrm{DOR})$ were 0.96 $(0.87,1.06)$ and $0.94(0.83,1.06)$, respectively. GMRs $(90 \% \mathrm{CI})$ of $3 \mathrm{TC}$ and tenofovir $\mathrm{AUC}_{0-\infty}$ $(\mathrm{DOR}+3 \mathrm{TC}+\mathrm{TDF} / 3 \mathrm{TC}+\mathrm{TDF})$ were $0.94(0.88,1.00)$ and $1.11(0.97,1.28)$, respectively Study drugs were generally well tolerated.

Conclusions: Multiple doses of TDF did not have a clinically meaningful effect on DOR PK. The PK of DOR were similar when administered alone or in combination with 3TC and TDF. DOR had no meaningful effect on the PK of 3TC and tenofovir. 


\section{INTRODUCTION}

Human immunodeficiency virus (HIV) continues to be a major global health challenge, infecting more than 36.9 million people worldwide [1]. In 2017, approximately 1.8 million people became infected with HIV, and 0.9 million people died from acquired immunodeficiency syndrome (AIDS)-related causes globally [1]. Antiretroviral therapy (ART) has been seminal in reducing the morbidity and mortality associated with HIV type 1 (HIV-1) infection. There are over 25 agents available for use in seven major mechanistic classes of ARTs: nucleoside reverse transcriptase inhibitors (NRTIs), non-nucleoside reverse transcriptase inhibitors (NNRTIs), protease inhibitors, a fusion inhibitor, a C-C chemokine receptor type 5 antagonist, a CD4directed post-attachment inhibitor, and integrase strand transfer inhibitors [2]. Current guidelines generally recommend three antiviral agents from at least two different mechanistic classes, as treatment with agents across classes has demonstrated sustained virologic response [2, 3]. Despite the array of therapies currently available, no single antiviral agent or combination of agents is appropriate for every person living with HIV, and there are often additional challenges in finding the most suitable treatment including issues with resistance, tolerability, unfavourable drug-drug interaction (DDI) profiles, high pill burden and/or unfavourable dosing frequency [2, 3].

NNRTIs were formerly the cornerstone of front-line therapy; however, as protease inhibitors and integrase strand transfer inhibitors offer a greater barrier to resistance, improved tolerability and more rapid viral suppression for people living with HIV, they are no longer primarily recommended in major international guidelines [2,3]. Although the NNRTIs efavirenz and rilpivirine remain as alternative treatment options under particular clinical circumstances, efavirenz has a relatively high rate of central nervous system-related adverse events (AEs), 
limiting its tolerability; and rilpivirine has lower virological efficacy, particularly in patients with high baseline HIV-1 RNA (>100,000 copies/ml) and low CD4+ T-cell counts $\left(<200\right.$ cells $\left./ \mathrm{mm}^{3}\right)$ [2]. As such, an unmet medical need exists for improved ART, including new NNRTI agents with improved tolerability and efficacy compared with currently available drugs in this class.

Doravirine (DOR, MK-1439) is a novel NNRTI designed to overcome the common resistance mutations which can reduce the effectiveness of other antiretrovirals in this class. Preclinical studies have demonstrated DOR to be active against wild-type HIV-1, as well as the two most prevalent NNRTI-associated mutant viruses (K103N and Y181C substitutions) [4]. In two Phase III studies, DOR demonstrated robust and durable efficacy, and was generally well tolerated [5, 6]. In the first of these, DOR co-administered with lamivudine (3TC)/tenofovir disoproxil fumarate (TDF) was associated with fewer treatment-emergent central nervous system AEs compared with the combination of efavirenz and emtricitabine/TDF[6]. In the second study, which compared DOR to ritonavir-boosted darunavir when both were co-administered with investigator-selected NRTIs (TDF and emtricitabine or abacavir and 3TC), there were no clinically relevant differences in the incidence of specific AEs, with the exception of a higher incidence of diarrhoea in the darunavir group [5]. In both studies, DOR combination therapy was associated with a more favourable lipid profile and similar antiviral efficacy over 48 weeks of treatment $[5,6]$. DOR $100 \mathrm{mg}$ administered once daily is indicated for the treatment of HIV-1 infection in combination with other ARTs, including 3TC and TDF, and is available for use as a single tablet or in a fixed dose combination tablet with 3TC and TDF [7, 8].

DOR is cleared primarily by oxidative metabolism via cytochrome P450 (CYP)3A [9]. Thus, drugs that induce or inhibit CYP3A may affect DOR elimination; this interaction has been confirmed in clinical DDI studies with the antibiotics rifabutin and rifampin, the antifungal 
ketoconazole, and the antiretrovirals ritonavir and efavirenz [10-14]. DOR was also shown to be a substrate for P-glycoprotein (P-gp) [9]; however, studies conducted to date revealed that P-gp does not have a significant role in DOR absorption or elimination, suggesting that the likelihood of P-gp affecting DOR pharmacokinetics (PK) is minimal [9]. In vitro studies demonstrated that DOR is not expected to have a meaningful impact on the PK of other compounds, including substrates of all major CYPs and drug transporter [15]. Clinical drug-interaction studies with CYP3A and transporter substrates demonstrated no substantive interactions [16-19].

As a commonly used NRTI with a well-characterized PK profile, 3TC is eliminated primarily via urinary excretion by active organic cationic secretion and is not a known perpetrator of DDIs [20]. TDF is another commonly used NRTI which, following absorption, is rapidly converted to its active metabolite, tenofovir, and cleared by renal elimination [21, 22]. Although tenofovir has been shown to reduce CYP1A substrate concentrations, it is not a substrate, inducer or inhibitor of CYP3A [21]. Tenofovir DDIs have been reported with didanosine, resulting in increased didanosine concentrations after co-administration [23] and with ritonavir-boosted and unboosted atazanavir, with co-administration resulting in decreased atazanavir plasma concentrations and increased tenofovir concentration $[21,24]$.

Based on the metabolic profiles of DOR, 3TC and TDF, a meaningful PK DDI is unlikely. However, due to the use of these three agents in combination, and the unexpected effects seen with TDF when co-administered with other antiretroviral agents, two clinical trials were conducted to further explore potential DDIs. 


\section{METHODS}

\section{Study design}

Study 1 (protocol MK-1439-003) was an open-label, two-period, fixed-sequence study in 8 healthy male participants, conducted between 19 September and 23 November 2011. In Period 1, all participants received a single oral dose of DOR $100 \mathrm{mg}$ after an overnight fast. After a washout of $\geq 7$ days, Period 2 began; all participants received a daily dose of TDF $300 \mathrm{mg}$ for 18 days with co-administration of a single dose of DOR $100 \mathrm{mg}$ on day 14. All doses of TDF alone were administered within 30 minutes prior to or after a standard meal; on day 14, study drugs were co-administered in the fasted state.

Study 2 (protocol MK-1439-038) was an open-label, single-dose, randomized, three-period crossover study in 15 healthy participants, conducted in January 2015. In the three treatment periods, participants received the following in a randomized manner: (A) a single oral dose of DOR $100 \mathrm{mg}$; (B) co-administration of single oral doses of 3TC $300 \mathrm{mg}$ and TDF $300 \mathrm{mg}$; and (C) co-administration of single oral doses of DOR $100 \mathrm{mg}$, 3TC $300 \mathrm{mg}$ and TDF $300 \mathrm{mg}$. Study drugs were administered after an overnight fast. The washout period between drug administrations was $\geq 7$ days.

The studies were conducted in accordance with principles of Good Clinical Practice and were approved by the appropriate institutional review boards (Study 1: Thomas Jefferson University IRB, Philadelphia, PA, USA; Study 2: the Institutional Review Board of Optimum Clinical Research Inc., Oshawa, Ontario, Canada) and regulatory agencies. 


\section{Study populations}

Study 1 included healthy men, $18-50$ years of age with a body mass index $\leq 35 \mathrm{~kg} / \mathrm{m}^{2}$. Study 2 included healthy men and women, $18-65$ years of age with a body mass index of $19-33 \mathrm{~kg} / \mathrm{m}^{2}$. In both studies, participants with a history of clinically significant medical conditions, estimated creatinine clearance of $\leq 80 \mathrm{ml} / \mathrm{min}$ (based on Cockcroft-Gault equation), drug or alcohol abuse, recent smoking or positive test for HIV, or who were hepatitis B or C positive, were excluded. Concomitant medications were not permitted from 14 days or 5 half-lives prior to the start of the trials until trial completion (although participants could receive concomitant therapy and continue in the study if the sponsor and investigator agreed). Participants in both studies provided written, informed consent prior to any study-related procedures being performed.

\section{Sample collection and plasma concentration determination}

In Study 1, blood samples for assay of DOR plasma concentration were obtained pre-dose and up to $120 \mathrm{~h}$ following administration of DOR on day 1 (Period 1), and co-administration of DOR and TDF on day 14 (Period 2). In Study 2, blood samples were collected pre-dose and up to $72 \mathrm{~h}$ post-dose.

In both studies, DOR plasma concentrations were analysed by liquid-liquid extraction for analyte isolation followed by liquid chromatographic-tandem mass spectrometric (LC-MS/MS) detection using a validated method (MSD, Oss, Netherlands) [13]. The lower limit of quantitation was $1 \mathrm{ng} / \mathrm{ml}$. The analytical range of the assay was $1.00-1,000 \mathrm{ng} / \mathrm{ml}$. For Study 1 , the inter-day accuracy of the quality control samples was $103.3-105.0 \%$, and the inter-day precision was 3.3-5.2\%. For Study 2, the inter-day accuracy was 97.0-99.5\%, and the inter-day precision was 3.5-5.3\%. In Study 2, following extraction, the plasma concentrations of 3TC and 
tenofovir were determined by validated achiral LC-MS/MS detection methods (Pharma Medica Research, Inc., Mississauga, Ontario, Canada). The analytical ranges of the assays were 5.00$3,000 \mathrm{ng} / \mathrm{ml}$ for $3 \mathrm{TC}$ and $2.00-500 \mathrm{ng} / \mathrm{ml}$ for tenofovir. For 3TC, the inter-day accuracy of the quality control samples was $97.8-105.2 \%$, and the inter-day precision was $0.9-3.3 \%$. For tenofovir, the inter-day accuracy was $98.5-101.5 \%$, and the inter-day precision was $1.0-2.2 \%$.

\section{PK evaluations}

In Study 1, DOR area under the concentration-time curve from time 0 extrapolated to infinity $\left(\mathrm{AUC}_{0-\infty}\right)$, maximum plasma concentration $\left(\mathrm{C}_{\max }\right)$, time to reach maximum plasma concentration $\left(\mathrm{T}_{\max }\right)$ and the apparent terminal half-life $\left(\mathrm{t}_{1 / 2}\right.$, calculated as the quotient of the natural $\log$ of 2 (ln [2]) and apparent terminal elimination rate constant) were calculated using Phoenix ${ }^{\circledR}$

WinNonlin ${ }^{\circledR}$ (Version 6.3, Certara, Princeton, NJ, USA). The observed plasma concentrations at 24 h post-dose $\left(\mathrm{C}_{24 \mathrm{~h}}\right)$ were obtained directly from plasma concentrations using SAS (Version 9.3; SAS Institute Inc., Cary, NC, USA). In Study 2, values of the same PK parameters as in Study 1 were calculated for DOR, 3TC and tenofovir using the non-compartmental approach in Phoenix ${ }^{\circledR}$ WinNonlin ${ }^{\circledR}$.

\section{Safety and tolerability}

Safety and tolerability were assessed in both studies by physical examinations, vital signs, laboratory assessments and AE monitoring.

\section{Statistics}

In both studies, the individual values of $\mathrm{AUC}_{0-\infty}, \mathrm{C}_{\max }$ and $\mathrm{C}_{24 \mathrm{~h}}$ were $\mathrm{ln}$-transformed prior to analysis and evaluated separately using a linear mixed-effect model. In Study 1, treatment was a 
fixed effect and subject was a random effect. A two-sided 90\% CI for the geometric mean ratio (GMR; DOR + TDF / DOR alone) was generated for DOR $\mathrm{AUC}_{0-\infty}, \mathrm{C}_{\max }$ and $\mathrm{C}_{24 \mathrm{~h}}$ from the mixed-effect model. Tenofovir PK were not analysed. Descriptive statistics were provided for $\mathrm{T}_{\max }$ and apparent $\mathrm{t}_{1 / 2}$. Median values were reported for $\mathrm{T}_{\max }$ while the geometric mean was reported for $\mathrm{t}_{1 / 2}$.

In Study 2, $\mathrm{AUC}_{0-\infty}, \mathrm{C}_{\max }$ and $\mathrm{C}_{24 \mathrm{~h}}$ were analysed using a linear mixed-effect model appropriate for a three-period, two-treatment crossover design with fixed-effect terms for treatment and period. An unstructured covariance matrix was used to allow for unequal treatment variances and to model the correlation between different treatment measurements within the same subject via the REPEATED statement SAS PROC MIXED. Kenward and Roger's method was used to calculate the denominator degrees of freedom for the fixed effects (DDFM=KR).

A two-sided 90\% CI for the GMRs (DOR + 3TC + TDF / DOR alone) was generated for DOR $\mathrm{AUC}_{0-\infty}, \mathrm{C}_{\max }$ and $\mathrm{C}_{24 \mathrm{~h}}$.

In addition, 95\% CIs were generated from the above mixed-effect model for geometric means by treatment for DOR $\mathrm{AUC}_{0-\infty}, \mathrm{C}_{\max }$ and $\mathrm{C}_{24 \mathrm{~h}}$. $3 \mathrm{TC}$ and tenofovir $\mathrm{AUC}_{0-\infty}, \mathrm{C}_{\max }$ and $\mathrm{C}_{24 \mathrm{~h}}$ after coadministration of DOR $100 \mathrm{mg}$, 3TC $300 \mathrm{mg}$ and TDF $300 \mathrm{mg}$ were analysed in a similar manner.

\section{RESULTS}

\section{Study populations}

A total of 8 healthy male participants were enrolled in Study 1; one participant discontinued on day 11 in Period 2 due to an AE that was not study-drug related. A total of 15 participants were 
enrolled in Study 2, all of whom completed the study. Demographics for participants from both studies are summarized in Table 1.

\section{PK evaluations}

Study 1: mean plasma concentration-time curves for DOR alone or co-administered after multiple doses of TDF are shown in Figure 1A. DOR PK summary statistics are listed in Table 2. The GMRs (90\% CI) of DOR AUC $0_{0-\infty}$ and $\mathrm{C}_{24 \mathrm{~h}}$ (DOR + TDF / DOR alone) were 0.95 (0.80, $1.12)$ and $0.94(0.78,1.12)$, respectively. The GMR $(90 \% \mathrm{CI})$ of DOR $\mathrm{C}_{\max }$ was $0.80(0.64$, 1.01). $\mathrm{T}_{\max }$ and apparent $\mathrm{t}_{1 / 2}$ were similar between the two treatment groups.

Study 2: the mean plasma concentration-time profiles for DOR, 3TC and tenofovir following $\mathrm{DOR}$ or $3 \mathrm{TC}+\mathrm{TDF}$ administration or DOR $+3 \mathrm{TC}+\mathrm{TDF}$ co-administration are shown in Figure 1B-D. DOR, 3TC and tenofovir PK summary statistics are listed in Tables 2 and 3. The GMRs $(90 \% \mathrm{CI})$ of DOR $\mathrm{AUC}_{0-\infty}$ and $\mathrm{C}_{\max }(\mathrm{DOR}+3 \mathrm{TC}+\mathrm{TDF} / \mathrm{DOR}$ alone) were $0.96(0.87,1.06)$ and $0.97(0.88,1.07)$, respectively. The GMRs $(90 \% \mathrm{CI})$ of $3 \mathrm{TC}^{\mathrm{AUC}} \mathrm{C}_{0-\infty}$ and $\mathrm{C}_{\max }(\mathrm{DOR}+3 \mathrm{TC}$ + TDF / 3TC + TDF) were $0.94(0.88,1.00)$ and $0.92(0.81,1.05)$, respectively. GMRs $(90 \% \mathrm{CI})$ of tenofovir $\mathrm{AUC}_{0-\infty}$ and $\mathrm{C}_{\max }(\mathrm{DOR}+3 \mathrm{TC}+\mathrm{TDF} / 3 \mathrm{TC}+\mathrm{TDF})$ were $1.11(0.97,1.28)$ and $1.17(0.96,1.42)$, respectively. Individual PK ratios and corresponding GMR plots of DOR, 3TC and tenofovir with and without co-administration of companion agents are shown in Figure 2.

\section{Safety}

All treatment combinations were generally well tolerated. There were no serious AEs, events of clinical interest or deaths reported during the studies. All AEs were mild in intensity, of limited duration and resolved by the end of the study. 
In Study 1, 3 of the 8 participants (37.5\%) reported a total of 10 AEs, 4 of which were considered to be related to study treatment (fatigue, [DOR alone], headache [DOR alone], rash $[\mathrm{TDF}]$ and somnolence $[\mathrm{DOR}+\mathrm{TDF}])$. Headache was the only AE reported by more than one participant. One participant was discontinued on day 11 of Period 2 due to an AE that was not study-drug related.

In Study 2, 5 of the 15 participants (33.3\%) reported a total of 5 AEs. One incidence of somnolence (DOR alone) and one of headache (DOR + 3TC + TDF) were considered to be related to study treatment.

\section{DISCUSSION}

There is a continuing need for improved therapeutics for the treatment of HIV-1 infection. DOR is a novel HIV-1 NNRTI that is indicated for use in combination with other antiretroviral agents, and as a fixed-dose regimen with 3TC and TDF as a complete regimen, for the treatment of HIV1 infection in adults with no prior antiretroviral treatment history [7, 8]. Although the metabolic profiles of these agents do not suggest that there would be meaningful DDIs with coadministration, clinical investigation was pursued to further evaluate potential DDIs.

Data from the two studies reported here demonstrate that neither co-administration of multiple doses of TDF nor co-administration with single doses of 3TC + TDF (at the recommended therapeutic dose of $300 \mathrm{mg}$ each for $3 \mathrm{TC}$ and TDF) have a clinically meaningful impact on DOR PK. This is evidenced by a lack of a meaningful effect on DOR $\mathrm{AUC}_{0-\infty}, \mathrm{C}_{\max }$ and $\mathrm{C}_{24 \mathrm{~h}}$, with $\mathrm{AUC}_{0-\infty}$ and $\mathrm{C}_{24 \mathrm{~h}}$ GMRs close to unity and $\mathrm{C}_{\max }$ reduced by $20 \%$ following multiple doses of TDF. The minor reduction in DOR $\mathrm{C}_{\max }$ is not anticipated to have any meaningful impact on DOR efficacy or safety, as a DOR Phase IIb trial demonstrated similar efficacy to efavirenz 
across a range of doses from 25 to $200 \mathrm{mg}$ [25]. The single-dose DDI assessment in Study 2 further supports a lack of interaction, with DOR $\mathrm{AUC}_{0-\infty}, \mathrm{C}_{\max }$ and $\mathrm{C}_{24 \mathrm{~h}}$ all without clinically meaningful changes.

3TC and TDF are commonly administered together, without evidence of a meaningful interaction when co-administered [20, 21]. As such, Study 2 was designed with coadministration of $3 \mathrm{TC}+\mathrm{TDF}$ without evaluation of each of the separate components. Data showed the lack of a meaningful effect of DOR on either 3TC or tenofovir PK. Tenofovir exposure and $\mathrm{C}_{\max }$ increased slightly (by $11 \%$ and $17 \%$, respectively) with co-administration of DOR. These changes are not clinically meaningful, based on drug-interaction effects and dosing recommendations for TDF [21]. The cause of the effect is unknown. It has been noted that tenofovir is a substrate of P-gp and breast cancer-resistant protein (BCRP) transporters [21]. Atazanavir and other HIV protease inhibitors, which are P-gp and BCRP inhibitors, modestly increase tenofovir plasma concentrations, although not to a clinically meaningful level, likely secondary to P-gp and BCRP inhibition [21, 26, 27]. However, in vitro observations with DOR have shown that it is not an inhibitor of P-gp, indicating that interactions between DOR and P-gp are unlikely to be the cause of the increases to plasma tenofovir levels in the current study [15].

Study 1 was designed to assess the impact of TDF at steady state on DOR PK to maximize any potential inductive or time-dependent effects of TDF. While Study 2 was conducted with singledose administration only, no inductive effects by DOR, 3TC or tenofovir were anticipated and there is no time dependence for the PK of DOR, 3TC or tenofovir. Moreover, with Study 1 data demonstrating minimal effect of TDF on DOR PK, a single-dose assessment was considered an appropriate approach for Study 2 and is anticipated to be predictive of multiple-dose behaviour [28]. The results of these studies did not demonstrate any substantive effect and indicate that, 
with multiple-dose administration, there would not be a meaningful PK DDI between these agents.

Administration of DOR, 3TC and TDF individually and in combination was generally well tolerated, providing further evidence of the tolerability of DOR alone and in combination with 3TC and TDF. The most common treatment-related AEs in both Study 1 and Study 2 were headache and somnolence, which have also been reported in DOR Phase III studies [5, 6]. The lack of DDIs between DOR and 3TC + TDF supports the fixed-dose, three-drug, single-tablet regimen (MK-1439A [DOR $100 \mathrm{mg} / 3 \mathrm{TC} 300 \mathrm{mg} / \mathrm{TDF} 300 \mathrm{mg}$ ]) that has been developed [29], and which was the formulation used in a recently reported Phase III study (discussed in the Introduction) [6].

In summary, multiple doses of TDF co-administered with a single dose of DOR did not have a clinically meaningful effect on the PK of DOR. DOR, 3TC and tenofovir PK were similar when administered alone or co-administered. Consequently, co-administration of the three drugs without dose adjustment is supported.

\section{ACKNOWLEDGEMENTS}

The authors would like to thank the trial staff and participants. In addition, the authors would like to thank Paul Fackler and Marty Behm for their help with the study. Medical writing and editorial assistance, under the direction of the authors, was provided by Annette Smith, $\mathrm{PhD}$, of CMC AFFINITY, a division of Complete Medical Communications Ltd, Macclesfield, UK, in accordance with Good Publication Practice (GPP3) guidelines. This assistance was funded by Merck Sharp \& Dohme Corp., a subsidiary of Merck \& Co., Inc., Kenilworth, NJ, USA. 


\section{DISCLOSURE STATEMENT}

This research was funded by Merck Sharp \& Dohme Corp., a subsidiary of Merck \& Co., Inc., Kenilworth, NJ, USA. JG, LF, KLY, IT and MI are employees of Merck Sharp \& Dohme Corp., a subsidiary of Merck \& Co., Inc., Kenilworth, NJ, USA, and may own stock and/or hold stock options in Merck \& Co., Inc., Kenilworth, NJ, USA. MSA, CR, YG and RL were employees of Merck Sharp \& Dohme Corp., a subsidiary of Merck \& Co., Inc., Kenilworth, NJ, USA at the time the study was conducted. WKK has no conflicts of interest to disclose. 


\section{REFERENCES}

1. UNAIDS. Global HIV \& AIDS Statistics - 2018 Fact Sheet. 2018. (Accessed 14 May 2019.) Available from: http://www.unaids.org/en/resources/fact-sheet.

2. Panel on Antiretroviral Guidelines for Adults and Adolescents. Guidelines for the Use of Antiretroviral Agents in Adults and Adolescents Living with HIV. 2018. (Accessed 14 May 2019.) Available from: https://aidsinfo.nih.gov/contentfiles/lvguidelines/adultandadolescentgl.pdf.

3. European AIDS Clinical Society. European guidelines for treatment of HIV-positive adults in Europe, version 9.1. 2018. (Accessed 14 May 2019.) Available from: http://www.eacsociety.org/files/2018_guidelines-9.1-english.pdf.

4. Lai MT, Feng M, Falgueyret JP, et al. In vitro characterization of MK-1439, a novel HIV-1 nonnucleoside reverse transcriptase inhibitor. Antimicrob Agents Chemother 2014; 58:1652-1663.

5. Molina JM, Squires K, Sax PE, et al. Doravirine versus ritonavir-boosted darunavir in antiretroviral-naive adults with HIV-1 (DRIVE-FORWARD): 48-week results of a randomised, doubleblind, phase 3, non-inferiority trial. Lancet HIV 2018; 5:e211-e220.

6. Orkin C, Squires KE, Molina JM, et al. Doravirine/Lamivudine/Tenofovir Disoproxil Fumarate is Non-inferior to Efavirenz/Emtricitabine/Tenofovir Disoproxil Fumarate in Treatment-naive Adults With Human Immunodeficiency Virus-1 Infection: Week 48 Results of the DRIVE-AHEAD Trial. Clinical infectious diseases : an official publication of the Infectious Diseases Society of America 2019; 68:535544.

7. Merck Sharp \& Dohme Corp. PIFELTRO (doravirine) Prescribing Information. Merck \& Co., Inc., Whitehouse Station, NJ, USA. 2018. (Accessed 8 March 2019.) Available from: https://www.merck.com/product/usa/pi_circulars/p/pifeltro/pifeltro_pi.pdf.

8. Merck Sharp \& Dohme Corp. DELSTRIGO (doravirine, lamivudine, and tenofovir disoproxil fumarate) Prescribing Information. Merck \& Co., Inc., Whitehouse Station, NJ, USA. 2018. (Accessed 8 March 2019.) Available from: https://www.merck.com/product/usa/pi_circulars/d/delstrigo/delstrigo_pi.pdf.

9. Sanchez RI, Fillgrove KL, Yee KL, et al. Characterisation of the absorption, distribution, metabolism, excretion and mass balance of doravirine, a non-nucleoside reverse transcriptase inhibitor in humans. Xenobiotica 2019; 49:422-432.

10. Khalilieh SG, Yee KL, Sanchez RI, et al. Multiple Doses of Rifabutin Reduce Exposure of Doravirine in Healthy Subjects. J Clin Pharmacol 2018; 58:1044-1052.

11. Anderson MS, Chung C, Tetteh E, et al. Effect of Ketoconazole on the Pharmacokinetics of Doravirine (MK-1439), a Novel Non-Nucleoside Reverse Transcriptase Inhibitor for the Treatment of HIV-1 Infection. Rev Antivir Ther Infect Dis. 2015, 16th International Workshop on Clinical Pharmacology of HIV \& Hepatitis Therapy, Washington, DC, USA.

12. Khalilieh S, Anderson M, Laethem T, et al. Multiple-Dose Treatment With Ritonavir Increases the Exposure of Doravirine. Conference on Retroviruses and Opportunistic Infections (CROI), February 13-16, 2017: Seattle, WA, USA. 2017. 
13. Yee KL, Khalilieh SG, Sanchez RI, et al. The Effect of Single and Multiple Doses of Rifampin on the Pharmacokinetics of Doravirine in Healthy Subjects. Clin Drug Investig 2017; 37:659-667.

14. Yee KL, Sanchez RI, Auger P, et al. Evaluation of Doravirine Pharmacokinetics When Switching from Efavirenz to Doravirine in Healthy Subjects. Antimicrob Agents Chemother 2017:61:e01757-01716.

15. Bleasby K, Fillgrove KL, Houle R, et al. In vitro evaluation of the drug interaction potential of doravirine. Antimicrob Agents Chemother 2019; 63:e2492-02418.

16. Anderson MS, Gilmartin J, Cilissen C, et al. Safety, tolerability and pharmacokinetics of doravirine, a novel HIV non-nucleoside reverse transcriptase inhibitor, after single and multiple doses in healthy subjects. Antivir Ther 2015; 20:397-405.

17. Anderson MS, Kaufman D, Castronuovo P, et al. Effect of doravirine (MK-1439) on the pharmacokinetics of an oral contraceptive (ethinyl estradiol [EE] and levonorgestrel [LNG]). International Workshop on HIV \& Women (IWHIVW). 2016, February 20-21, 2016, Boston, MA, USA.

18. Anderson MS, Khalilieh S, Yee KL, et al. A Two-Way Steady-State Pharmacokinetic Interaction Study of Doravirine (MK-1439) and Dolutegravir. Clin Pharmacokinetics 2017; 56:661-669.

19. Khalilieh S, Yee KL, Sanchez RI, et al. Results of a Doravirine-Atorvastatin Drug-Drug Interaction Study. Antimicrob Agents Chemother 2017:61:e01364-01316.

20. GlaxoSmithKline/ViiV Healthcare. Epivir (lamivudine) Prescribing Information. 2018. (Accessed 8 March 2019.) Available from:

https://www.gsksource.com/pharma/content/dam/GlaxoSmithKline/US/en/Prescribing_Information/Epivi r/pdf/EPIVIR-PI-PIL.PDF.

21. Gilead Sciences Inc. Viread (tenofovir disproxil fumarate) Prescribing Information. 2018. (Accessed 8 March 2019.) Available from: http://www.gilead.com/ /media/Files/pdfs/medicines/liverdisease/viread/viread_pi.pdf.

22. Kearney BP, Flaherty JF, Shah J. Tenofovir disoproxil fumarate: clinical pharmacology and pharmacokinetics. Clinical pharmacokinetics 2004; 43:595-612.

23. Kearney BP, Sayre JR, Flaherty JF, Chen SS, Kaul S, Cheng AK. Drug-drug and drug-food interactions between tenofovir disoproxil fumarate and didanosine. J Clin Pharmacol 2005; 45:13601367.

24. Taburet AM, Piketty C, Chazallon C, et al. Interactions between atazanavir-ritonavir and tenofovir in heavily pretreated human immunodeficiency virus-infected patients. Antimicrob Agents Chemother 2004; 48:2091-2096.

25. Gatell JM, Morales-Ramirez JO, Hagins DP, et al. Forty-eight-week efficacy and safety and early CNS tolerability of doravirine (MK-1439), a novel NNRTI, with TDF/FTC in ART-naive HIV-positive patients. J Int AIDS Soc 2014; 17:19532.

26. Gupta A, Zhang Y, Unadkat JD, Mao Q. HIV Protease Inhibitors Are Inhibitors but Not Substrates of the Human Breast Cancer Resistance Protein (BCRP/ABCG2). J Pharmacol Exp Ther 2004; 310:334-341.

27. Storch $\mathrm{CH}$, Theile D, Lindenmaier H, Haefeli WE, Weiss J. Comparison of the inhibitory activity of anti-HIV drugs on P-glycoprotein. Biochem Pharmacol 2007; 73:1573-1581. 
28. FDA. Clinical Drug Interaction Studies - Study Design, Data Analysis, and Clinical Implications. Guidance for Industry. 2017. (Accessed 10 July 2018.) Available from: https://www.fda.gov/downloads/drugs/guidances/ucm292362.pdf.

29. Behm MO, Yee KL, Liu R, Levine V, Panebianco D, Fackler P. The Effect of Food on Doravirine Bioavailability: Results from Two Pharmacokinetic Studies in Healthy Subjects. Clin Drug Investig 2017; 37:571-579. 


\section{DISPLAY ITEMS}

Table 1. Study population demographics

\begin{tabular}{|c|c|c|}
\hline Characteristic & $\begin{array}{l}\text { Study } 1 \\
(N=8)\end{array}$ & $\begin{array}{l}\text { Study } 2 \\
(N=15)\end{array}$ \\
\hline \multicolumn{3}{|l|}{ Gender, n (\%) } \\
\hline Male & $8(100)$ & $7(46.7)$ \\
\hline Female & 0 & $8(53.3)$ \\
\hline \multicolumn{3}{|l|}{ Age (years) } \\
\hline Mean (range) & $44.3(36-50)$ & $44(23-56)$ \\
\hline \multicolumn{3}{|l|}{ Body mass index $\left(\mathrm{kg} / \mathrm{m}^{2}\right)$} \\
\hline Mean ( \pm standard deviation) & $29.2 \pm 2.0$ & $26.2 \pm 2.9$ \\
\hline \multicolumn{3}{|l|}{ Race, $\mathbf{n}(\%)$} \\
\hline Asian & 0 & $2(13.3)$ \\
\hline Black or African American & $6(75.0)$ & $3(20.0)$ \\
\hline White & $2(25.0)$ & $10(66.7)$ \\
\hline
\end{tabular}


Table 2. Plasma PK of DOR $100 \mathrm{mg}$ when administered alone, with multiple-dose TDF $300 \mathrm{mg}$ administered once daily for 14 days, or with single-dose TDF $300 \mathrm{mg}$ and 3 TC $300 \mathrm{mg}$ to healthy participants

\begin{tabular}{|c|c|c|c|c|c|c|c|c|c|}
\hline \multirow{2}{*}{ PK parameter } & \multicolumn{3}{|c|}{ DOR + TDF } & \multicolumn{3}{|c|}{ DOR } & \multicolumn{3}{|c|}{ DOR + TDF / DOR } \\
\hline & $\mathbf{N}$ & GM & $95 \% \mathrm{CI}$ & $\mathbf{N}$ & GM & $95 \% \mathrm{CI}$ & GMR & $90 \% \mathrm{CI}$ & rMSE $^{a}$ \\
\hline $\operatorname{AUC}_{0-\infty}(\mathrm{h} \cdot \mu \mathrm{M})^{b}$ & 7 & 33.4 & $25.9,43.2$ & 8 & 35.3 & $27.5,45.3$ & 0.95 & $0.80,1.12$ & 0.162 \\
\hline $\mathrm{C}_{24 \mathrm{~h}}(\mathrm{nM})^{b}$ & 7 & 547 & 430,697 & 8 & 584 & 463,738 & 0.94 & $0.78,1.12$ & 0.171 \\
\hline $\mathrm{C}_{\max }(\mathrm{nM})^{b}$ & 7 & 1,310 & $965,1,780$ & 8 & 1,630 & $1,210,2,190$ & 0.80 & $0.64,1.01$ & 0.216 \\
\hline $\mathrm{T}_{\max }(\mathrm{h})^{c}$ & 7 & 3.0 & $1.0,7.9$ & 8 & 2.5 & $0.5,5.0$ & & & \\
\hline $\mathrm{t}_{1 / 2}(\mathrm{~h})^{d}$ & 7 & 15.4 & 25.0 & 8 & 14.4 & 24.7 & & & \\
\hline
\end{tabular}

Study 2: DOR administered with single-dose TDF and 3TC

\begin{tabular}{|c|c|c|c|c|c|c|c|c|c|}
\hline \multirow{2}{*}{ PK parameter } & \multicolumn{3}{|c|}{$\mathrm{DOR}+3 \mathrm{TC}+\mathrm{TDF}$} & \multicolumn{3}{|c|}{ DOR } & \multicolumn{3}{|c|}{ DOR + 3TC + TDF / DOR } \\
\hline & $\mathbf{N}$ & GM & $95 \% \mathrm{CI}$ & $\mathbf{N}$ & GM & $95 \% \mathrm{CI}$ & GMR & $90 \% \mathrm{CI}$ & Intra-subject $\% \mathrm{CV}^{e}$ \\
\hline $\operatorname{AUC}_{0-\infty}(\mathrm{h} \cdot \mu \mathrm{M})^{b}$ & 15 & 37.7 & $28.7,49.4$ & 15 & 39.1 & $31.5,48.6$ & 0.96 & $0.87,1.06$ & 15.2 \\
\hline $\mathrm{C}_{24 \mathrm{~h}}(\mathrm{nM})^{b}$ & 15 & 507 & 332,774 & 15 & 541 & 390,750 & 0.94 & $0.83,1.06$ & 19.6 \\
\hline $\mathrm{C}_{\max }(\mathrm{nM})^{b}$ & 15 & 2,030 & $1,720,2,400$ & 15 & 2,090 & $1,810,2,420$ & 0.97 & $0.88,1.07$ & 15.1 \\
\hline $\mathrm{T}_{\max }(\mathrm{h})^{c}$ & 15 & 2.0 & $1.0,6.0$ & 15 & 3.0 & $1.0,4.0$ & & & \\
\hline $\mathrm{t}_{1 / 2}(\mathrm{~h})^{d}$ & 15 & 13.5 & 40.6 & 15 & 13.8 & 31.9 & & & \\
\hline
\end{tabular}

${ }^{a}$ rMSE (residual error) from the linear mixed-effect model. When multiplied by 100 approximates the within-subject $\% \mathrm{CV}$ on the raw scale. ${ }^{b} \mathrm{Back}$-transformed

LSM and CI from linear mixed-effects model performed on natural log-transformed values. ${ }^{c}$ Median (minimum, maximum) reported for $\mathrm{T}_{\text {max }}{ }^{d} \mathrm{GM}$ and $\%$ geometric $\mathrm{CV}$ reported for $\mathrm{t}_{1 / 2}$. ${ }^{\mathrm{e}} \mathrm{Estimated}$ based on the elements of the variance-covariance matrix as: $\mathrm{CV}(\%)=100^{*} \mathrm{sqrt}\left[\left(\sigma \mathrm{A}^{2}+\sigma \mathrm{B}^{2}-2 * \sigma \mathrm{AB}\right) / 2\right] .3 \mathrm{TC}$,

lamivudine; $\mathrm{AUC}_{0-\infty}$, area under the concentration-time curve from time 0 to infinity; $\mathrm{C}_{\max }$, maximum plasma concentration; $\mathrm{C}_{24 \mathrm{~h}}$, concentration of analyte in plasma $24 \mathrm{~h}$ after administration; CI, confidence interval; CV, coefficient of variation; DOR, doravirine; GM, geometric mean; GMR, geometric mean ratio;

LSM, least-squares mean; PK, pharmacokinetic; $r M S E$, root mean square error; $\mathrm{t}_{1 / 2}$, apparent elimination half-life; $\mathrm{T}_{\text {max }}$, time to reach maximum plasma concentration; TDF, tenofovir disoproxil fumarate. 
Table 3. Plasma PK of 3TC and tenofovir following single-dose administration of 3 TC $300 \mathrm{mg}+\mathrm{TDF} 300 \mathrm{mg}$ or DOR $100 \mathrm{mg}+3 \mathrm{TC}$ $300 \mathrm{mg}+$ TDF $300 \mathrm{mg}$ to healthy participants

\begin{tabular}{|c|c|c|c|c|c|c|c|c|c|}
\hline \multicolumn{10}{|l|}{ 3TC } \\
\hline \multirow[b]{2}{*}{ PK parameter } & \multicolumn{3}{|c|}{ DOR + 3TC + TDF } & \multicolumn{3}{|c|}{$3 T C+T D F$} & \multicolumn{3}{|c|}{ DOR + 3TC + TDF / 3TC + TDF } \\
\hline & $\mathbf{N}$ & GM & $95 \% \mathrm{CI}$ & $\mathbf{N}$ & GM & $95 \% \mathrm{CI}$ & GMR & 90\% CI for GMR & Intra-subject $\% \mathrm{CV}^{a}$ \\
\hline $\operatorname{AUC}_{0-\infty}(\mathrm{h} \cdot \mathrm{ng} / \mathrm{ml})^{b}$ & 15 & 14,200 & $12,400,16,200$ & 15 & 15,000 & $13,800,16,500$ & 0.94 & $0.88,1.00$ & 9.7 \\
\hline $\mathrm{C}_{\max }(\mathrm{ng} / \mathrm{ml})^{b}$ & 15 & 2,910 & $2,460,3,450$ & 15 & 3,150 & $2,760,3,600$ & 0.92 & $0.81,1.05$ & 19.4 \\
\hline $\mathrm{T}_{\max }(\mathrm{h})^{c}$ & 15 & 1.00 & $1.0,2.0$ & 15 & 1.00 & $0.5,2.0$ & & & \\
\hline $\mathrm{t}_{1 / 2}(\mathrm{~h})^{d}$ & 15 & 15.9 & 57.6 & 15 & 15.7 & 32.2 & & & \\
\hline \multicolumn{10}{|l|}{ Tenofovir } \\
\hline & \multicolumn{3}{|c|}{ DOR + 3TC + TDF } & \multicolumn{3}{|c|}{ 3TC + TDF } & \multicolumn{3}{|c|}{ DOR + 3TC + TDF / 3TC + TDF } \\
\hline PK parameter & $\mathbf{N}$ & GM & $95 \% \mathrm{CI}$ & $\mathbf{N}$ & GM & $95 \% \mathrm{CI}$ & GMR & 90\% CI for GMR & Intra-subject $\% \mathbf{C V}^{a}$ \\
\hline $\mathrm{AUC}_{0-\infty}(\mathrm{h} \cdot \mathrm{ng} / \mathrm{ml})^{b}$ & 15 & 2,790 & $2,470,3,150$ & 15 & 2,500 & $2,090,2,990$ & 1.11 & $0.97,1.28$ & 20.5 \\
\hline $\mathrm{C}_{\max }(\mathrm{ng} / \mathrm{ml})^{b}$ & 15 & 338 & 286,400 & 15 & 289 & 237,352 & 1.17 & $0.96,1.42$ & 29.7 \\
\hline $\mathrm{T}_{\max }(\mathrm{h})^{c}$ & 15 & 1.0 & $0.5,2.0$ & 15 & 1.00 & $0.5,1.0$ & & & \\
\hline $\mathrm{t}_{1 / 2}(\mathrm{~h})^{d}$ & 15 & 20.9 & 18.5 & 15 & 19.7 & 12.6 & & & \\
\hline
\end{tabular}

${ }^{a}$ Estimated based on the elements of the variance-covariance matrix as: $\mathrm{CV}(\%)=100 * \operatorname{sqrt}\left[\left(\sigma \mathrm{A}^{2}+\sigma \mathrm{B}^{2}-2 * \sigma \mathrm{AB}\right) / 2\right] .{ }^{b} \mathrm{Back}$-transformed LSM and CI from linear mixed-effects model performed on natural log-transformed values. ${ }^{c}$ Median (minimum, maximum) reported for $\mathrm{T}_{\max }{ }^{d} \mathrm{GM}$ and $\%$ geometric $\mathrm{CV}$ reported for $\mathrm{t}_{1 / 2}$. 3TC, lamivudine; $\mathrm{AUC}_{0-\infty}$, area under the concentration-time curve from time 0 to infinity; $\mathrm{C}_{\max }$, maximum plasma concentration; $\mathrm{CI}$, confidence interval;

$\mathrm{CV}$, coefficient of variation; DOR, doravirine; GM, geometric mean; GMR, geometric mean ratio; LSM, least-squares mean; PK, pharmacokinetic; $\mathrm{t}_{1 / 2}$, apparent elimination half-life; $\mathrm{T}_{\max }$, time to reach maximum plasma concentration; $\mathrm{TDF}$, tenofovir disoproxil fumarate. 
Figure 1. Arithmetic mean ( \pm standard deviation) plasma concentration-time profiles of (A) single-dose DOR $100 \mathrm{mg}$ alone and co-administered with TDF $300 \mathrm{mg}$ after 14 days of once-daily TDF administration ( $N=8$, inset=semi-log scale); (B) single-dose DOR $100 \mathrm{mg}$ alone and co-administered with single doses of 3TC $300 \mathrm{mg}+$ TDF $300 \mathrm{mg}$; (C) single-dose 3TC 300 mg following administration of 3TC $300 \mathrm{mg}+$ TDF $300 \mathrm{mg}$ and DOR $100 \mathrm{mg}+3 \mathrm{TC} 300 \mathrm{mg}+$ TDF $300 \mathrm{mg}$ and (D) tenofovir following administration of 3TC $300 \mathrm{mg}+$ TDF $300 \mathrm{mg}$ and DOR $100 \mathrm{mg}+3$ TC $300 \mathrm{mg}+$ TDF $300 \mathrm{mg}(N=15$, inset=log-linear scale for B, C and D)

A

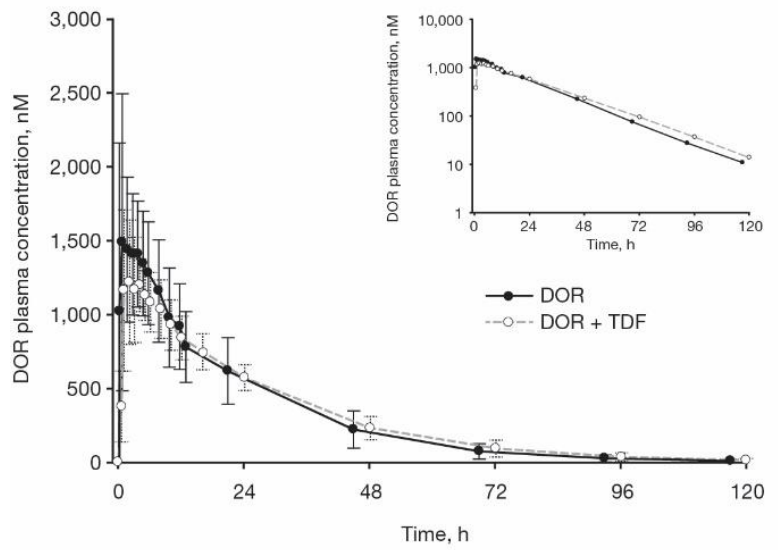

C

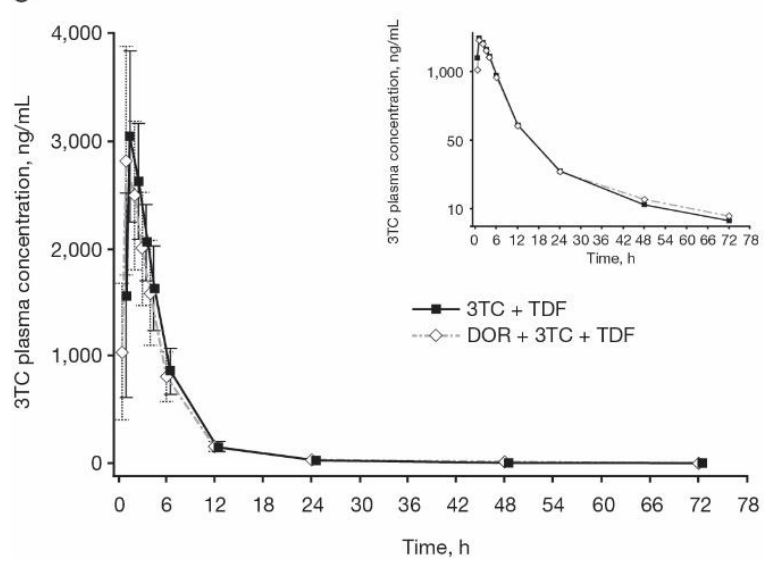

B

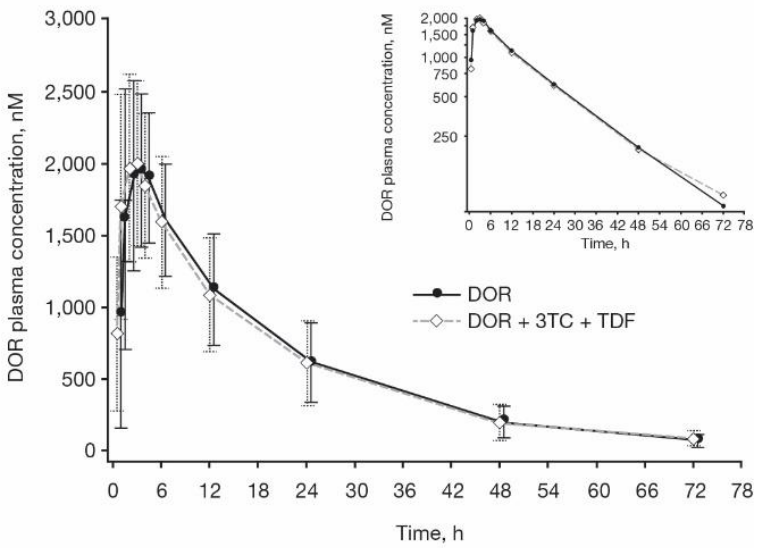

D

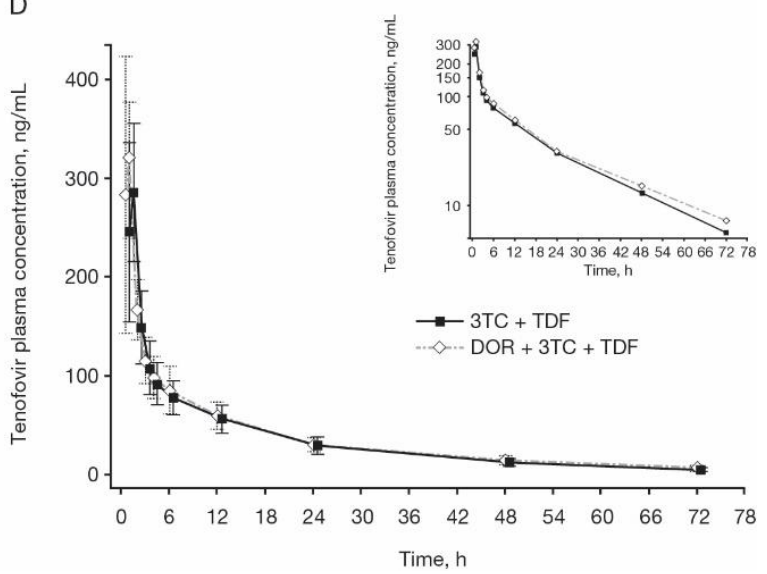

3TC, lamivudine; DOR, doravirine; h, hours; TDF, tenofovir disoproxil fumarate. 
Figure 2. Individual plasma pharmacokinetic ratios and corresponding geometric mean ratios with $90 \%$ confidence intervals for (A) single-dose DOR $100 \mathrm{mg}$ with and without multiple-dose TDF $300 \mathrm{mg}(n=8)$, (B) single-dose DOR $100 \mathrm{mg}$ with and without single-dose 3TC $300 \mathrm{mg}+$ TDF $300 \mathrm{mg}(n=15)$, (C) 3TC after administration of single-dose 3TC $300 \mathrm{mg}$ + TDF $300 \mathrm{mg}$, with DOR $100 \mathrm{mg}$ versus without DOR $(n=15)$ and (D) tenofovir after administration of 3TC $300 \mathrm{mg}$ + TDF $300 \mathrm{mg}$, with DOR $100 \mathrm{mg}$ versus without DOR $(n=15)$

A

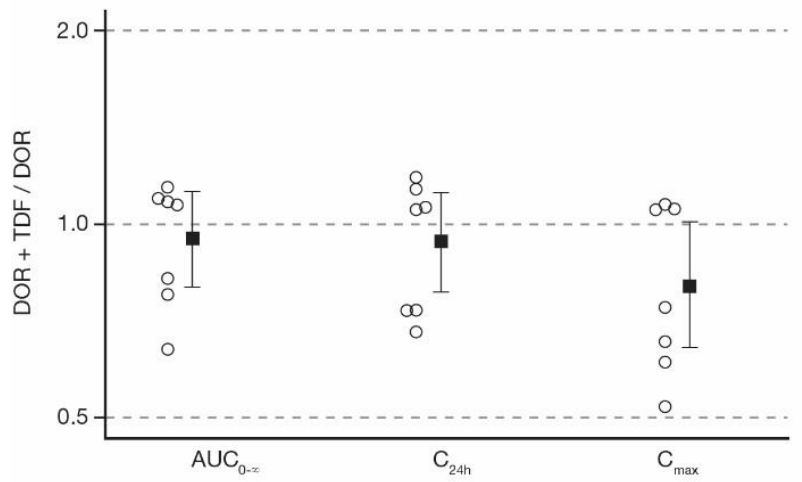

C

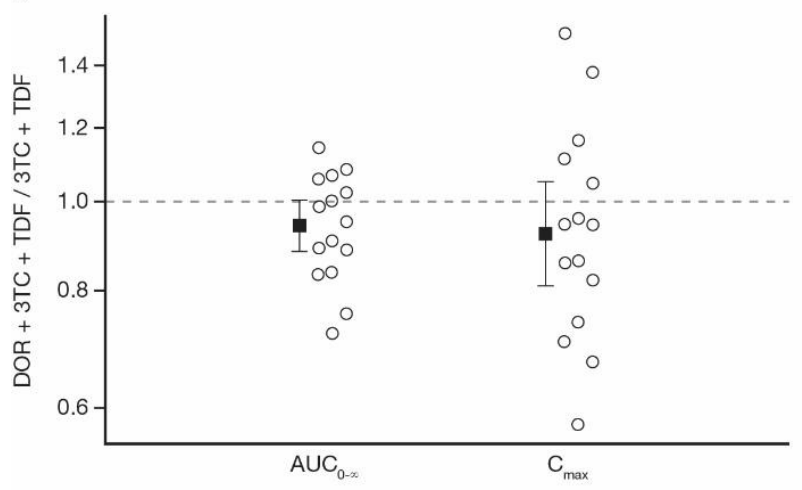

B

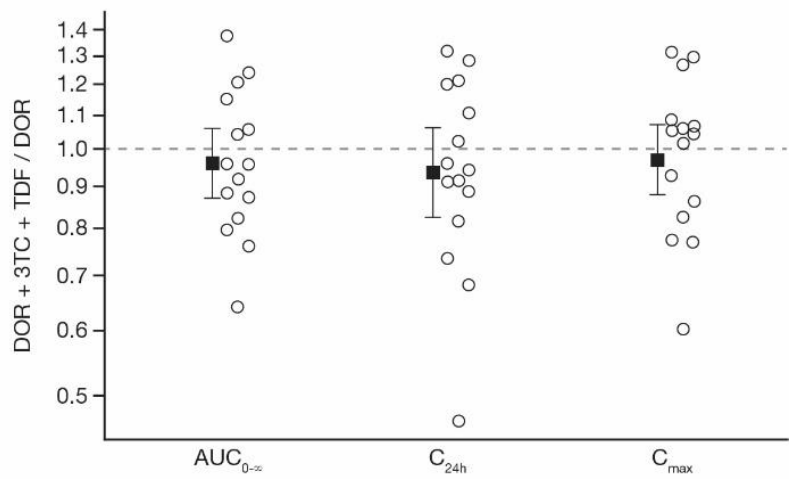

D

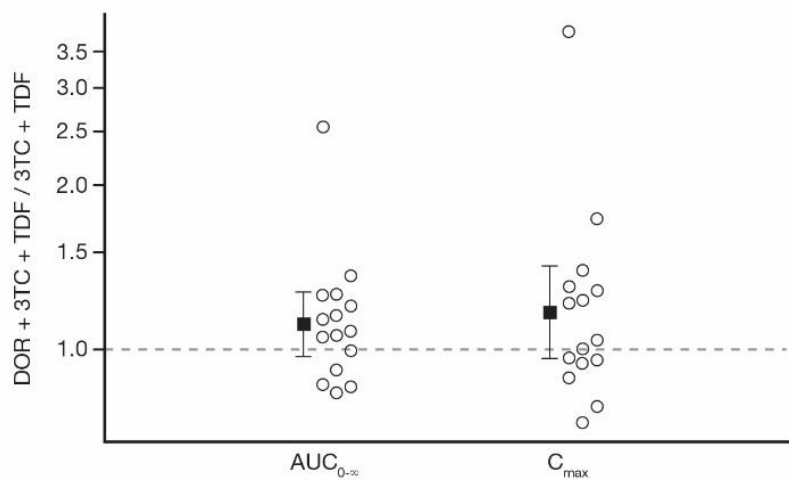

O Individual ratios a GMR with $90 \% \mathrm{Cl}$

3TC, lamivudine; $\mathrm{AUC}$, area under the concentration-time curve; $\mathrm{AUC}_{0-\infty}, \mathrm{AUC}$ from time 0 to infinity; $\mathrm{C}_{\max }$, maximum concentration; $\mathrm{C}_{24}$, concentration of analyte in plasma $24 \mathrm{~h}$ after administration; DOR, doravirine; TDF, tenofovir disoproxil fumarate. 